\title{
SOME INEQUALITIES CONCERNING FUNCTIONS OF EXPONENTIAL TYPE
}

\author{
BY R. J. DUFFIN AND A. C. SCHAEFFER
}

In this paper we derive two inequalities concerning entire functions satisfying the conditions $f(z)=O\left(e^{n|z|}\right)$, and $|f(z)| \leqq 1$ on the real axis. Our results are closely related to theorems given by S. Bernstein, Szegö, van der Corput and Schaake, and Boas.*

THEOREM I. Let $f(z)$ be an entire function such that

$$
f(z)=O\left(e^{n|z|}\right)
$$

uniformly over the entire plane, and on the real axis

$$
|f(z)| \leqq 1 \text {. }
$$

Then, if $a$ and $b$ are any two real numbers,

$$
\left|a f(z)+b f^{\prime}(z)\right| \leqq\left(a^{2}+n^{2} b^{2}\right)^{1 / 2}
$$

for real $z$.

Proof of Theorem I : By means of Cauchy's Integral Formula and the Phragmén-Lindelöf Principle it is not difficult to show that if $f(z)$ satisfies the conditions of Theorem I then

$$
\frac{d}{d z}\left\{\frac{f(z)}{\sin n(z+\alpha)}\right\}=-n \sum_{k=-\infty}^{\infty} \frac{(-1)^{k} f\left(\frac{k \pi}{n}-\alpha\right)}{\{n(z+\alpha)-k \pi\}^{2}} .
$$

For the proof of (4) see Pólya and Szegö, Aufgaben und Lehrsätze aus der Analysis, vol. II, page 35 and page 218. If $\alpha$ is real we have $|f(k \pi / n-\alpha)| \leqq 1$, hence for all real $z$ and $\alpha$

$$
\begin{aligned}
\mid f^{\prime}(z) \sin n(z+\alpha)- & n f(z) \cos n(z+\alpha) \mid \\
& \leqq \sum_{-\infty}^{\infty} \frac{n \sin ^{2} n(z+\alpha)}{\{n(z+\alpha)-k \pi\}^{2}} .
\end{aligned}
$$

* S. Bernstein, Comptes Rendus, vol. 176 (1923), p. 1603.

Van der Corput and Schaake, Compositio Matematica, vol. 2 (1935), p. 321; vol. 3 (1936), p. 128.

Szegö, Schriften der Königsberger gelehrten Gesellschaft, Naturwissenschaftliche Klasse, Fünftes Jahr 4 (1928), p. 69.

Boas, Transactions of this Society, vol. 40 (1936), p. 287. 
Using the expansion $1 / \sin ^{2} n w=\sum_{-\infty}^{\infty} 1 /(n w-k \pi)^{2}$, we obtain

$$
\left|f^{\prime}(z) \sin n(z+\alpha)-n f(z) \cos n(z+a)\right| \leqq n \text {. }
$$

Given any real numbers $a$ and $b$ we may choose an $\alpha$ such that $\sin n(z+\alpha)=b /\left(a^{2} / n^{2}+b^{2}\right)^{1 / 2}$ and $-n \cos n(z+\alpha)$ $=a /\left(a^{2} / n^{2}+b^{2}\right)^{1 / 2}$. Substitution in (6) then proves Theorem I.

THEOREM II. Let $f(z)$ be a function which is real on the real axis and which satisfies the conditions of Theorem I. Then, if $z=x+i y$,

$$
\left|f^{\prime}(z)\right|^{2}+n^{2}|f(z)|^{2} \leqq n^{2} \cosh 2 n y .
$$

Unless $f(z)$ is of the form $\cos n(z+\alpha)$ the equality sign can occur only at points on the real axis where $f= \pm 1$.

Proof of Theorem II : If $n(z+\alpha)$ is not a multiple of $\pi$ the equality sign can occur in (5) only if $\pm f(k \pi / n-\alpha)=(-1)^{k}$ for all $k$. Putting these values for $f(k \pi / n-\alpha)$ in (4) one finds $f(z) \equiv \pm \cos n(z+\alpha)$. If $n(z+\alpha)$ is a multiple of $\pi$ the equality sign in (5) clearly can occur only if $f(z)= \pm 1$.

In (3) let $a=n^{2} f$ and $b=f^{\prime}$. Then we find for real $z$

$$
\left\{f^{\prime}(z)\right\}^{2}+n^{2}\{f(z)\}^{2} \leqq n^{2}
$$

We shall suppose throughout that $f(z)$ is not of the form $\cos n(z+\alpha)$ for any real $\alpha$. Then the equality sign can occur in (8) only at points where $f(z)= \pm 1$. From (8) we have $\left|f^{\prime}(z)\right|<n$ for real $z$. It is easy to show that $f^{\prime}(z)=O\left(e^{n|z|}\right)$, so that $f^{\prime}(z) / n$ satisfies all the conditions that $f(z)$ does, and we have from (8)

$$
\left\{\frac{f^{\prime \prime}(z)}{n}\right\}^{2}+n^{2}\left\{\frac{f^{\prime}(z)}{n}\right\}^{2}<n^{2}
$$

This is a strict inequality, for the equality can occur only at points where $f^{\prime}(z) / n= \pm 1$, and we have shown that $\left|f^{\prime}(z)\right|<n$.

In the same way one shows by induction that for the higher derivatives

$$
\left\{\frac{f^{(k+1)}(z)}{n^{k}}\right\}^{2}+n^{2}\left\{\frac{f^{(k)}(z)}{n^{k}}\right\}^{2}<n^{2} .
$$

Thus if $f(z)$ is expanded in a power series

$$
f(z)=\sum_{\nu=0}^{\infty} \frac{a_{\nu}}{\nu !} z^{\nu},
$$


we have $n^{2} a_{\nu}^{2}+a_{\nu+1}^{2} \leqq n^{2(\nu+1)}$, or

$$
\left|n a_{\nu}+i a_{\nu+1}\right| \leqq n^{\nu+1} \text {. }
$$

The equality sign can occur only if $\nu=0$.

Let us write $f(z)$ in the form

$$
f(z)=g(z)+u(z)=\sum_{0}^{\infty} \frac{a_{2 \nu}}{(2 \nu) !} z^{2 \nu}+\sum_{0}^{\infty} \frac{a_{2 \nu+1}}{(2 \nu+1) !} z^{2 \nu+1}
$$

where $g(z)$ is even and $u(z)$ is odd. We see that on the imaginary axis $(z=i y)$ the functions $g(z)$ and $u^{\prime}(z)$ are real while $g^{\prime}(z)$ and $u(z)$ are pure imaginary. Let $|y|>0$. Then

$$
\begin{aligned}
n^{2}|f(i y)|^{2}+\left|f^{\prime}(i y)\right|^{2}=n^{2} \mid & \left.g(i y)\right|^{2}+n^{2}|u(i y)|^{2} \\
& +\left|g^{\prime}(i y)\right|^{2}+\left|u^{\prime}(i y)\right|^{2} .
\end{aligned}
$$

Combining the two even functions $g$ and $u^{\prime}$ we obtain

$$
\begin{aligned}
n^{2}|g(i y)|^{2}+\left|u^{\prime}(i y)\right|^{2} & =\left|n g(i y)+i u^{\prime}(i y)\right|^{2} \\
& =\left|\sum_{0}^{\infty} \frac{n a_{2 \nu}+i a_{2 \nu+1}}{(2 \nu) !}(i y)^{2 \nu}\right|^{2} .
\end{aligned}
$$

By the inequality (10) this is less than

$$
\left|\sum_{0}^{\infty} \frac{n^{2 \nu+1}}{(2 \nu) !} y^{2 \nu}\right|^{2}=n^{2} \cosh ^{2} n y .
$$

Combining the odd functions $u$ and $g^{\prime}$, we have

Thus we have

$$
\begin{aligned}
n^{2}|u(i y)|^{2}+\left|g^{\prime}(i y)\right|^{2} & =\left|n u(i y)+i g^{\prime}(i y)\right|^{2} \\
& =\left|\sum_{0}^{\infty} \frac{n a_{2 \nu+1}+i a_{2 v+2}}{(2 \nu+1) !}(i y)^{2 \nu+1}\right|^{2} \\
& <\left|\sum_{0}^{\infty} \frac{n^{2 \nu+2}}{(2 \nu+1) !} y^{2 \nu+1}\right|^{2}=n^{2} \sinh ^{2} n y .
\end{aligned}
$$

$n^{2}|f(i y)|^{2}+\left|f^{\prime}(i y)\right|^{2}<n^{2} \cosh ^{2} n y+n^{2} \sinh ^{2} n y=n^{2} \cosh 2 n y$.

We have thus demonstrated that (7) holds on the imaginary axis; but if $\beta$ is real $f(z+\beta)$ also satisfies the conditions, so this is sufficient. We remark that if $f(z)=\cos n(z+\alpha)$, (7) becomes an equality throughout the plane.

PuRdue University 